\title{
A Survey on: Detection from Attacks on Web Application using IDS Approaches
}

\author{
Radha Rashmi Tiwari \\ Truba College \\ RGPV University, Bhopal
}

\author{
Amit Saxena \\ Truba College \\ RGPV University, Bhopal
}

\begin{abstract}
Currently, digital time provides more opportunities to the users, contains applications of web which can be used for own purposes at workplace, school, lectures and more. At present, several aspects promoted with the application on web otherwise are the major problem encountered in cyber-attacks. This document gives detail of the IDS which generally stored applications and to generate a warning whenever find all sorts of attacks. This work, IDS uses these functions packets of networks or string properties of inputs which are again selected relevant for some attacks analysis. Maintaining functions is time consuming aa well as requires a low security rating. Moreover, Accompanying some of the helpful techniques which are quite helpful in classifying behaviors, which are often costly as well as robust for production, require large information for bedtime and application demand. This contribution contributes towards exploring autonomous intruder detection systems.
\end{abstract}

\section{Keywords}

Web security, Intrusion Detection System, Robustness

\section{INTRODUCTION}

The total no. hacking and invasion increases by one year whenever any new technology come into existence. Guaranteeing the dimension exceptionally high security and dependable correspondence of data between various associations is turning into a significant issue. Therefore, the IDS has transformed into a basic portion in PC and framework security [1]. An IDS is a contraption or programming thing that separates the moving toward traffic on the framework for a wrong development (or interruption) and triggers a ready when harmful attack is distinguished. The inspiration driving IDS is the usage of unlawful and furthermore ill-advised framework by unapproved customers through the control of framework traffic and control data. An harmful attack can be described as a movement of exercises that endeavor to affirm the trustworthiness, mystery, or availability of advantages on the framework [2-5]. Respectability: Data honesty infers that data has not been improperly adjusted amid the movement or limit. Mindful measures contains the physical appearance of the systems just as the servers which don't enable the entrance to the information alongside its upkeep more extensive availability practice. Classification: Confidential information is the information which isn't effectively open or approved to unapproved clients. Accessibility: Availability affirms that the frameworks are diminished in like manner also advertisement the clients are drafted on schedule, (for example, when clients required). Something contrary to accessibility is the refusal of administration, where clients don't approach the assets they as of now have. IDS offer two Methods to investigate the incorporation traffic: disappointment system and peculiarity strategy. Damaging IDs pursue well-characterized examples (or marks), which can normally be found by fitting examples to gathered types of preparing information. This cutoff points false positives, which ordinarily not all. With infection scanners, misuse based IDs can't decide director in system which can't think about them (I e New Attack). For maltreatment IDS which is helpful, its marks must guarantees the consistency refreshed. IDS center around inconsistencies contains surveys of typical framework client peculiarities. They are found by making an ordinary profile of the PC being controlled and perceiving significant transformations from that profile. Since, IDS setup contains a tight clarification of ordinary on an irregularity premise, it additionally produces a tremendous measure of false positives. The segment investigates the potential components of beginning profound learning with intrude on disclosure programs. Our methodology deep penetrates the factual substance and implemented, since the entire methodology to peak about what we talk for example Rough data is set in a framework specific an irregular loss of state. In this way, that there will be no commitment clients to choose specialists and develop propelled names for readiness.

\section{* Interruption Detection Approaches}

The four significant IDS name-HIDS, NIDS, MIDS, AB IDS. Each get-away methodology deals with the actualities of specific standards. The present area quickly portrays the motorization of every discovery group just as the related difficulties. Facilitated based IDS (HIDS): Host-put together IDS thinks about information with respect to has as hosts side. The host-based system engineering is specialist based.

This expresses each host facilitated by the PC is relegated a S/W operator. A HIDS just checks the gadget's inbound and outbound bundles and cautions the client or supervisor of suspicious action. System based IDS (NIDS): Intrusion discovery depends on the system in light of the fact that the framework is utilized to investigate organize parcels.

This is instead of host-based intrusion disclosure, which frames data that is made on the PC itself, for example, event and bit logs. Framework groups are commonly shut some place close to the framework, notwithstanding the way that they can be hindered by the yield of switches and switches. The most usually used show is TCP/IP. Framework resources are stand-out in that they are accessible to unauthenticated clients or outer clients.

They are set up to empower the availability and withdrawal of administrations from the system. Misuse based IDS: Abusebased IDS are additionally alluded to as signature-based IDS. Each occurrence in a record is set apart as should be expected or fizzled, and a learning issue calculation is prepared on the featured information.

These procedures can naturally change interruption danger models into various info information, which are new sorts of harmful attacks. For whatever length of time that they are 
checked as needs be. This technique explicitly utilizes known examples of unapproved conduct to give and epitomize consequent visits. These particular examples are called appropriations. For host-based revelation discovery, three fizzled logins are a case of a mark.

For system section ID, an imprint can be as direct as a particular model that organizes a portion of a framework parcel. For example, group or bundle content imprints or potentially header content imprints may exhibit unapproved exercises, for instance, invalid FTP exercises. The characteristic of an imprint can mean an unapproved get to which is normally visited. Contingent upon the maintainability and seriousness of the marked signature, a few cautions, answers or notices should be sent to the proper experts. They have an abnormal state of value in recognizing known harmful attacks and their variations. Their impediment is that they can't find obscure interlopers and search for endowments that are deferred by open undertakings.

Peculiarity based IDS: Anomaly-based IDSs have been created to create surprising examples of conduct. The IDS characterizes a standard of the general client ace and anything that is wide because of its width is set apart as a conceivable impression. What might be considered as irregularities may vary, however we as a rule consider an abnormality of all events that go amiss from the factual standard by pretty much than two standard frequencies. It recognizes variations from the norm as irregular conduct and consequently identifies a dismissal of the banner, the last one just as the confided in one. Not at all like endorsement based IDSs, these methods recognize new kinds of impressions and deviations from typical use. It's an extremely amazing and novel apparatus, however a potential moment is the exceptionally false alert.

Already obscure (yet genuine) framework controls can likewise be perceived as abnormalities and therefore set apart as checked. SQL Injection (SQLI) SQLI is a code infusion technique that maintains a strategic distance from security vulnerabilities in an application's database. It would appear that an harmful attack SQL catchphrase is being utilized as the information fragment. It works because of erroneous or poor verification of the info information.

In the event that you can utilize SQLI, wrong clients can increase unapproved rights to the database and play out the database control. Tragically, infusion harmful attacks can be isolated into three sorts. In first-request harmful attack, mappings or sub-SQL questions are gone into existing statements. In second-request harmful attacks, the shopping center code is constantly put away in the database. The aggressor endeavors to discover inner application clients, framework clients who use income, web search tools, etc. In Laterin Injection, the PL/SQL system can deal with harmful attacks so that even client input does not happen. It goes about as a variable that relates the information type or number with the SQL explanation content.

On the off chance that the flawed client finds an information cell, diverse SQLI types are utilized to execute harmful attacks of various kinds [2].

\section{LITERATURE SURVEY}

1) Blocking of SQL Injection Attacks by Comparing Static and Dynamic Queries (Jaskanwal Minhas and Raman Kumar) (2013) Minhas and Kumar suggested an unmistakable, convincing and successful system [6] to reveal SQLI strikes and depict and found another ambush other than existing SQLI strikes named void territory control ambush. In this suggestion, static and dynamic examination is joined. Making trademark characteristics analyzes static and dynamic SQL questions. The inquiries posed are trademark and static examinations with a similar number of illustrations to abbreviate the reaction time. In the wake of emptying the properties, SQL inquiries become autonomous, connecting this strategy to any database.

2) SQLStor: Block or Stored Procedure SQL Injection Attack with Dynamic Query Structure Validation (Sruthy Mamadhan, Manesh T, Varghese Paul) (2012) Mamadhan et al. In [7], another framework for exchanging SQL imbuements into JSP web applications was created. The thought relies upon the affirmation of the structure of the dynamic inquiry to the execution. For instance, the technique incorporates different establishment frameworks, for example For instance, initial an accidental inquiry from the main examination, trading the customer mediation for the question with delicate information sources. At that point survey the structure of the kind inquiry for endorsement. As of now, stacked issues are characterized in both unambiguous SQL look and non-malignant question building. On the off chance that the two qualities ??contrast, the SQL implantation approach is accessible as of now and can be put away from execution to the last semantic review. At the shot of being disappointed, cut bombs are worked for both the examination and the visits. At the shot of being equivalent, the inquiry is passed into the general harmful attack, which is tallied. This approach disallows various kinds of implantations.

3) SQLIMW: another device against SQL infusion (Gao Jiao, Xing Chang-Ming, JING Maohua) (2012) Jiao et al. [8] present a sinusoidal sintered instrument (SQLIMW). This strategy incorporates middleware to avert SQL infusion harmful attacks. SQLIMW requires all the more prosperity and adaptability by ascertaining HASH-empowered encryption while utilizing useable username, mystery language articulation, and SQLIMW private key for the XOR task. It applies to the standard course for encryption and references to the Hath system. With both encryption and remarkable data in the unwinding and decoding stages, the hash change execution arrange is quicker than DES and other cryptographic changes. This strategy isn't restricted to a solitary site level. It very well may be in any web application structure that contains data about the database.

4) Injection syringe clearing of SQL inquiry characteristics (Jeom-Goo Kim) (2011) Jeom-Goo Kim was proposed [9] for a perfect, powerful SQL question end framework that utilizes static and dynamic research. This framework takes a gander at and breaks down the post by discarding the property picture of SQL questions. It is normal that a limit $\mathrm{F}$ has the abilities to build up the element in sql questions. Responsibility for SQL question marks and runtime standard SQL checks are cleared. No assailant was observed to be at the point that even after the abrogation of conspiracy now, the issue equals the initial investment the fixed SQL question. In spite of the deviations remains dependably in an inquiry, an offer is being used for this time. The impediment of this need is which isn't gotten supply of the power at that point.

5) Sharp Interrupted Investigation associated with Innovations in Innovation Innovations (Chai Wenguuang, Tan Chunhui, and Duan Yuting) (2011) Wenguuang and Yuting [15] produce a splendid business advancement that utilizes data about mining. In this strategy, information in the site database is sent by data put away with the assistance of expert, sending information to find the ticket deals activity. The commencement of wellbeing is finished by a mindful 
evaluation that gives a notice for all distinguished harmful attack. The shrewd interference acknowledgment with web data mining is found more profitable than customary intrusion area structure Improved Information burrowing computations is required for overhaul of proposed framework.

\section{PROPOSED APPROACH}

Considering every frameworks examined its methodologies the rate for the risk factor interruption in powerless measures. The proposed technique completely follows web administration. Web governments deserve substantial work on the web applications. This work is quite specialized organization that distributes the accessible web administration for the customer's questions. Customers receive specific message through server database this site. Each customer is customized accordingly web administration. It is possible to elaborate the customer's all requests free web administration. Here discus the attacks distinguished dependent on mapping model among inquiries and predefined marks. Arrangement of every harmful attack is completed and need to take some actions which are quite preventive in nature are joined with limit Attacks.

\section{CONCLUSION}

This work shows different short letter with different security strategies was introduced. As per these publications, IDS is unable $100 \%$ in order to get security for applications, but provides security for exceptional measures. It has factors as accessible attack accuracy. This research is adjusted to application which are running strikes also the usage of other methods to eliminate such pauses.

\section{ACKNOWLEDGMENTS}

Our thanks to the experts who have contributed towards development of the template.

\section{REFERENCES}

[1] Bowman, M., Debray, S. K., and Peterson, L. L. 1993. Reasoning about naming systems.

[2] Ding, W. and Marchionini, G. 1997 A Study on Video Browsing Strategies. Technical Report. University of Maryland at College Park.

[3] Fröhlich, B. and Plate, J. 2000. The cubic mouse: a new device for three-dimensional input. In Proceedings of the SIGCHI Conference on Human Factors in Computing Systems

[4] Tavel, P. 2007 Modeling and Simulation Design. AK Peters Ltd.

[5] Sannella, M. J. 1994 Constraint Satisfaction and Debugging for Interactive User Interfaces. Doctoral Thesis. UMI Order Number: UMI Order No. GAX9509398., University of Washington.

[6] Forman, G. 2003. An extensive empirical study of feature selection metrics for text classification. J. Mach. Learn. Res. 3 (Mar. 2003), 1289-1305.

[7] Brown, L. D., Hua, H., and Gao, C. 2003. A widget framework for augmented interaction in SCAPE.

[8] Y.T. Yu, M.F. Lau, "A comparison of MC/DC, MUMCUT and several other coverage criteria for logical decisions", Journal of Systems and Software, 2005, in press.

[9] Spector, A. Z. 1989. Achieving application requirements. In Distributed Systems, S. Mullender. 